\title{
Effect of fluoxetine on the MAPK-ERK1/2 signaling pathway and expression of associated factors in human conjunctival epithelial cells in culture
}

\author{
TING CAO, YANNING YANG, BIN CHEN and XIAOXIONG WANG \\ Department of Ophthalmology, Renmin Hospital of Wuhan University, Wuhan, Hubei 430060, P.R. China
}

Received June 30, 2018; Accepted October 29, 2019

DOI: $10.3892 /$ etm.2020.9482

\begin{abstract}
The aim of the present study was to evaluate the effect of fluoxetine on activation of the mitogen-activated protein kinase (MAPK) signaling pathway and the expression of apoptosis-associated factors in human conjunctival epithelial cells (HConEpiCs) in culture. HConEpiCs were isolated, cultured and characterized by immunostaining. HConEpiC cells at passage 3-4 were cultured with fluoxetine at different dosages $(0,1,2.5,5,10,20$ and $40 \mu \mathrm{M})$ and proliferation rates were determined using a Cell Counting Kit-8 assay. Subsequently, Transwell assays were performed to evaluate the effect of fluoxetine $(5 \mu \mathrm{M})$ on the invasion and migration capacities of HConEpiCs. ERK1/2 and phosphorylated (p-)ERK1/2 levels were also evaluated in control and fluoxetine-treated groups of HConEpiCs via immunostaining. Finally, western blot assays were performed to evaluate the intracellular protein levels of ERK, p-ERK, Bcl-2, Bax and matrix metalloproteinases (MMPs) in HConEpiCs. It was identified that, as the fluoxetine concentration increased, proliferation rates of HConEpiCs gradually decreased and $5 \mu \mathrm{M}$ fluoxetine was selected for further evaluation. The results of Transwell assays indicated that fluoxetine treatment significantly repressed cell migration and invasion. Immunostaining suggested that there was no significant difference in fluorescence intensity of ERK1/2 between the control and fluoxetine-treated groups, while p-ERK1/2 was significantly enhanced in the fluoxetine-treated group. This result indicated that fluoxetine promoted ERK1/2 activation without affecting its expression. Similarly, western blot analysis revealed no significant difference in ERK1/2 and MMP levels between fluoxetine-treated and control groups, but p-ERK1/2 and Bax were upregulated and Bcl-2 was decreased in the fluoxetine-treated group. In conclusion, fluoxetine
\end{abstract}

Correspondence to: Dr Ting Cao, Department of Ophthalmology, Renmin Hospital of Wuhan University, 238 Jiefang Road, Wuhan, Hubei 430060, P.R. China

E-mail: caotingdoctor@yeah.net

Key words: dry eye disease, fluoxetine, mitogen-activated protein kinase induces apoptosis of HConEpiCs in culture via activating the MAPK-ERK1/2 signaling pathway.

\section{Introduction}

Dry eye syndrome is characterized by multifactorial tear film instability, which consequently triggers inflammation and damage to conjunctival epithelial cells (1). The expression levels of inflammatory factors, including interleukin (IL)-1 and tumor necrosis factor (TNF)- $\alpha$, are upregulated in dry eye syndrome. In turn, this activates the mitogen-activated protein kinase (MAPK) signaling pathway and induces a series of downstream changes, ultimately resulting in dry eyes $(2,3)$. The MAPK signaling pathway is crucial for signaling transduction in mammalian cells. Via the MAPK pathway, signals from extracellular stimuli maybe transduced into nuclei and are able to mediate cellular responses (4-8). There are several branches of the MAPK pathway, among which the MAPK-ERK pathway has a role in amplifying extracellular signals $(9,10)$.

Fluoxetine is a selective serotonin reuptake inhibitor (SSRI) that is widely administered as an anti-depressant drug. A previous study by the present group investigated the prevalence of dry eye diseases among patients suffering from depression from 2009 to 2015, and the results demonstrated that dry eye syndrome was significantly more common when patients received SSRI treatment. SSRIs exert their anti-depressant effect via regulating the MAPK-ERK signaling pathway, and may also trigger ocular surface inflammation and cellular apoptosis by elevating the expression of IL- $\beta$ and TNF- $\alpha$ via MAPK-ERK (11). This is a potential mechanism by which anti-depressant drugs promote dry eye syndrome. In this present study, it was speculated that the pathogenesis of dry eye syndrome caused by anti-depressant drugs may be due to SSRIs inhibiting 5-hydroxytryptamine (5-HT) reuptake by presynaptic membrane 5 -HT pumps in neurons, increasing the number of 5-HT receptors. This would activate the MAPK signaling pathway, increasing the levels of phosphorylated (p-)ERK, which may in turn induce an increase in matrix metalloproteinases (MMPs) and inflammatory factors and promote apoptosis. This would ultimately result in the symptom of dry eyes. However, the mechanisms by which an increase in 5-HT receptors activates the MAPK-ERK signaling pathway have remained elusive $(6,7,12)$. In the present study, cellular 
apoptosis was observed via the gene expression of $\mathrm{Bcl}-2$, Bax and MMPs in order to provide novel evidence for the mechanism by which anti-depressant drugs induce dry eye diseases.

\section{Materials and methods}

Materials. Human conjunctival epithelial cells (HConEpiCs) were derived from the primary culture of conjunctival tissue from healthy adult human donors, which was provided by the Eye Center of Renmin Hospital at Wuhan University (Wuhan, China), collected between January 2016 and October 2016. A total of 20 patients (10 males and 10 females) with ocular fundus diseases, aged from 20-45 years, requiring surgical treatment were recruited. They were required to have healthy ocular surface with no conjunctival lesions and no cardiovascular disease. The selected healthy adults were confirmed not to have dry eye syndrome. The standard of exclusion contained the following aspects: Tear break-up time (BUT>10 sec), tear river width $(>0.3 \mathrm{~mm})$ determined with an Ocular surface analyzer and Schirmer test 2 (>10 $\mathrm{mm}$ at $5 \mathrm{~min})$. Low-glucose Dulbecco's modified Eagle's medium (DMEM)was purchased from Boster Biological Technology Co., Ltd. Patient consent was obtained and ethical approval of the present study was granted by the Clinical Research Ethics Review Board of Wuhan University (Wuhan, China). The present study collected 20 cases of abandoned conjunctival tissues during the operations. Each tissue size was about $5 \times 5 \mathrm{~mm}$ and were stored at $4^{\circ} \mathrm{C}$ in sterile glass bottles. Trypsin-EDTA was manufactured by Shanghai Chemical Reagent Co., Ltd. Antibodies against Cytokeratin-13 (CK-13; cat. no. YM6162R) were purchased from Immunoway Biotechnology Company. Antibodies against Bax (cat. no. 9942), Bcl2 (cat. no. 9941), MMP2 (cat. no. 4022), MMP9 (cat. no. 3852) and GAPDH (cat. no. 5174) were purchased from Cell Signaling Technology (all 1:1,000). TRIzol ${ }^{\circledR}$ was produced by Invitrogen (Thermo Fisher Scientific, Inc.); fluoxetine was purchased from Sigma-Aldrich (Merck KGaA); Cell Counting Kit-8 (CCK-8) cell proliferation kits were purchased from Nanjing Jiancheng Bioengineering Institute; mouse-anti-human ERK1/2 monoclonal antibody (cat. no. 9926) and mouse-anti-human p-ERK1/2 monoclonal antibody (cat. no. 9910; both used at 1:1,000) were purchased from Upstate Biotechnology, Inc.; horseradish peroxidase (HRP)-conjugated goat anti-mouse secondary antibody was purchased from Boster Biological Technology Co., Ltd. (cat. no. BA1054); the enhanced chemiluminescence reagent kit was purchased from Pierce (Thermo Fisher Scientific, Inc.).

Culture and characterization of HConEpiCs. HConEpiCs were isolated for further culture using trypsin-EDTA mixture digestion solution. Conjunctival tissue was obtained from healthy adult human donors. The tissue was chopped into small pieces using scissors, followed by treatment with trypsin at $37^{\circ} \mathrm{C}$ for $10 \mathrm{~min}$. The trypsin digestion was neutralized by addition of medium. The tissue chunks were then further broken down by pipetting and the flakes of tissue were aspirated away. Subsequently, the cell suspension was centrifuged at $72 \times \mathrm{g}$ for $6 \mathrm{~min}$ at room temperature. The supernatant was removed and the cell density was adjusted to $4-6 \times 10^{4}$ cells $/ \mathrm{ml}$. The cells were seeded at $4-6 \times 10^{4}$ cells $/ \mathrm{ml}$ (a total of 50,000 cells) into culture flasks and cultured in an incubator at $5 \% \mathrm{CO}_{2}$ and $37^{\circ} \mathrm{C}$. The cells were cultured in low-glucose DMEM with 10\% FBS, (Gibco; Thermo Fisher Scientific, Inc.; cat. no. 10099) The medium was replaced every 3-4 days and the growth of the cells was closely monitored under an inverted light microscope. Cells were passaged after confluence was reached. Experiments were performed using cells at passages 2-4. The growth of the HConEpiCs was observed under an inverted microscope and images were captured. The expression profile of human conjunctival epithelium indicated that the most abundant gene transcript was that of CK-13 (13). Hence, CK-13 immunofluorescence detection was used to examine the cultured cells.

CCK-8 assay. HConEpiCs in the logarithmic phase were seeded in 96-well plates with $100 \mu \mathrm{l}$ medium per well and incubated with $5 \% \mathrm{CO}_{2}$ at $37^{\circ} \mathrm{C}$. After $24 \mathrm{~h}$ of incubation, fluoxetine at different dosages $(0,1,2.5,5,10,20$ and $40 \mu \mathrm{M})$ was added to the culture, followed by incubation for another $24 \mathrm{~h}$ at $37^{\circ} \mathrm{C}$. For each dose, three replicates were performed. The culture medium was removed and $100 \mu 1$ serum-free DMEM basal medium with $10 \mu \mathrm{l}$ CCK-8 was added to each well, and cells were further cultured for $2 \mathrm{~h}$. The integrated optical density (IOD) values were measured at $450 \mathrm{~nm}$ using an ELISA reader and converted into proliferation rates. Cell proliferation rates were calculated as (IOD $\left.{ }^{\text {untreated }}-\mathrm{IOD}^{\text {treated }}\right) / \mathrm{IOD}^{\text {untreated }} \times 100 \%$. The appropriate fluoxetine dose for subsequent experiments was selected according to the cell proliferation rates.

Transwell invasion and migration assays. For the invasion assay, $100 \mu \mathrm{l}$ serum-free DMEM containing $10 \mu \mathrm{l}$ Matrigel was added to the wells of Transwell chambers. The chambers were incubated for $3 \mathrm{~h}$ in an incubator to allow for the Matrigel to solidify. The following experimental groups were set up: Control group and $5 \mu \mathrm{M}$ fluoxetine group. The cells were re-suspended at $1 \times 10^{5} / \mathrm{ml}$ with serum-free DMEM. Subsequently, $200 \mu \mathrm{l}$ cell suspension was added to the Matrigel-coated wells and $500 \mu 1$ DMEM containing 20\% FBS was added to the lower chambers. After $24 \mathrm{~h}$ of incubation, cells were fixed with $4 \%$ paraformaldehyde (PFA) for $1 \mathrm{~h}$ at room temperature and stained with $0.25 \%$ crystal violet at the room temperature for $20 \mathrm{~min}$. Cells on the upper chamber of the transwell membrane were wiped away using a cotton swab and five fields were randomly selected for examination under an inverted microscope (magnification, x100). Images were obtained and the number of cells was counted. Six replicates were performed for each condition.

For the migration assay, the cells were divided into a control group and a $5 \mu \mathrm{M}$ fluoxetine group. Cells $\left(2 \times 10^{4}\right)$ were re-suspended in serum-free medium $24 \mathrm{~h}$ post-treatment and seeded into the top wells of the Transwell chambers, and $500 \mu \mathrm{l}$ of RPMI-1640 medium containing 20\% fetal bovine serum was added to the lower chambers. Samples were collected after $20 \mathrm{~h}$ of incubation, fixed with 4\% PFA for $20 \mathrm{~min}$ at room temperature and stained with $0.25 \%$ crystal violet for $20 \mathrm{~min}$ at room temperature. The cells in the upper chamber that did not pass through the matrix glue were wiped off, leaving the cells that passed through the membrane using a cotton swab and five fields were randomly selected for examination under an inverted microscope (magnification, x100). Images 

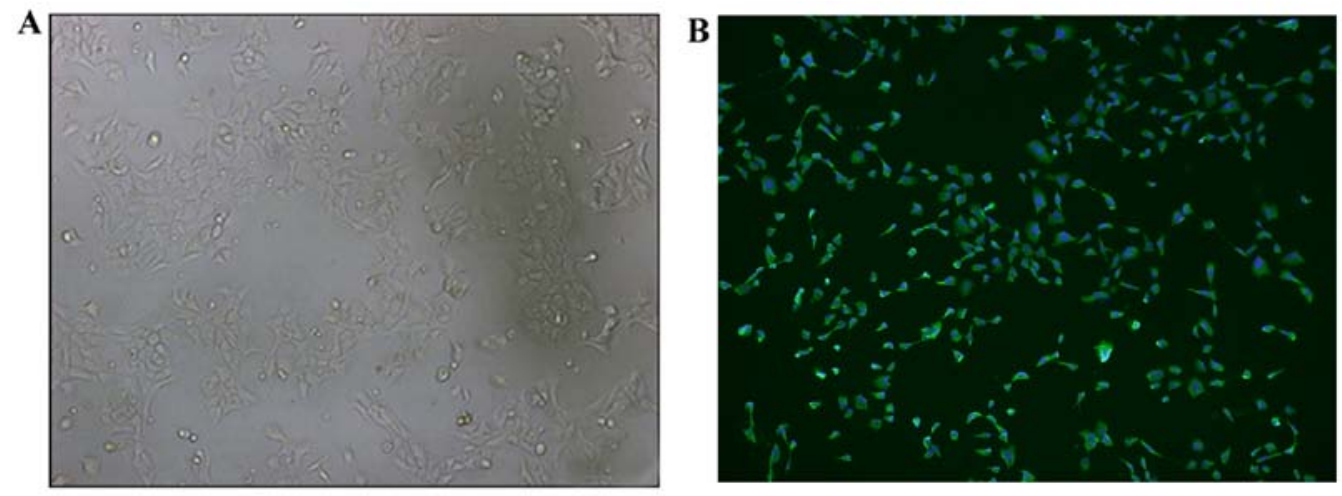

Figure 1. Images of HConEpiCs observed via inverted microscopy. (A) HConEpiCs were robustly growing in culture after passaging (magnification, x100). (B) Cells were positive for cytokeratin-13 (green; magnification, x100; nuclei were counterstained in blue using DAPI). HConEpiCs, human conjunctival epithelial cells.

were obtained and the number of cells was counted. For each condition, six replicates were performed.

Immunofluorescent staining for ERK1/2 and p-ERK1/2 in HConEpiCs. Cells in the logarithmic growth phase were seeded on gelatin-coated coverslips. The inoculation density was $1 \times 10^{4}$ cells $/ \mathrm{ml}$, and the cells were allowed to adhere for $24 \mathrm{~h}$ and fixed with 4\% PFA for $15 \mathrm{~min}$ at room temperature. The gelatin coating solution was purchased from Beijing Reagan Biotechnology Co., Ltd. (cat. no. IH0205). The fixed samples were washed three times with PBS for $5 \mathrm{~min}$ and permeabilized with $1 \%$ Triton-X-100 for $10 \mathrm{~min}$. The cells were blocked with $1 \%$ bovine serum albumin (Beyotime Institute of Biotechnology; cat. no. P0007) for $1 \mathrm{~h}$ at room temperature and incubated with primary antibody against ERK1/2 or p-ERK1/2 (1:100 dilution) overnight at $4^{\circ} \mathrm{C}$. The samples were then washed with PBS three times and incubated with HRP conjugated goat anti-rabbit secondary antibodies (1:100 dilution; Thermo Fisher Scientific, Inc.; cat. no. 31460) for $1 \mathrm{~h}$ at room temperature. The nuclei were counterstained with DAPI for $15 \mathrm{~min}$ and the cells were washed with PBS three times for $15 \mathrm{~min}$. Finally, the slides were covered with cover slips, sealed with glycerol and subjected to imaging using a fluorescence microscope (BX51; Olympus Corporation). The nuclei were visualized in blue and ERK1/2 or p-ERK1/2 were labeled with red fluorescence.

Western blot analysis. The protein levels of intracellular ERK, p-ERK, Bax, Bcl-2 and MMP-2/9 were evaluated. HConEpiCs were treated with a gradient of doses of fluoxetine $(0,1,2.5$ $, 5,10,20$ and $40 \mu \mathrm{M})$ for $24 \mathrm{~h}$ and total protein was then extracted. The Pierce ${ }^{\mathrm{TM}}$ BCA Protein Assay Kit (Thermo Fisher Scientific, Inc.; cat. no. 23227) was used to quantify protein levels. Protein samples $(20 \mu \mathrm{g})$ were mixed with loading buffer and loaded onto $10 \%$ separating gels and 5\% stacking gels for SDS-PAGE. The proteins were then transferred to a nitrocellulose membrane (Beyotime Institute of Biotechnology; cat. no. FFN08). The membrane was blocked with 5\% fat-free milk in PBS for $1 \mathrm{~h}$ at room temperature and incubated with primary antibodies overnight at $4^{\circ} \mathrm{C}$. The membrane was then washed in PBS three times and incubated with a HRP-conjugated secondary antibody $(1: 10,000$; Thermo Fisher Scientific, Inc.; cat. no. 31430) for $1 \mathrm{~h}$ at room

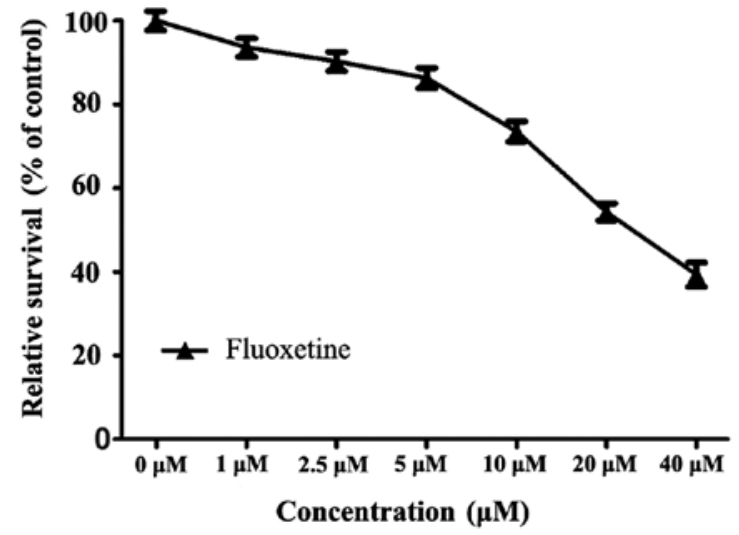

Figure 2. Viability curve of human conjunctival epithelial cells treated by a gradient of fluoxetine dosages for $24 \mathrm{~h}$. The relative viabilities were calculated as follows: Absorbance of treatment group/absorbance of control group $\mathrm{x} 100 \%$. The survival rate in the control group was set as $100 \%$.

temperature. After three rounds of membrane washing, enhanced chemiluminescence was performed to visualize the protein. The dilution the antibodies against ERK, p-ERK, Bax, Bcl-2 and MMP-2/9 was 1:1,000.

Statistical analysis. Statistical analysis was performed with SPSS12.0 (SPSS, Inc.). Values are expressed as the mean \pm standard error. Student's t-tests were used for comparisons between two groups. One-way ANOVAs were used for comparisons between multiple groups and post-hoc analysis was performed using Tukey's highly significant differences test.

\section{Results}

Isolation and characterization of primary HConEpiCs. It was observed via inverted microscopy that the recovered HConEpiCs started adhering to the culture dish and proliferating at $0.5 \mathrm{~h}$ post-cell seeding. HConEpiCs appeared morphologically round with clear cell membranes and nuclei in the middle of the cells. These cells started to migrate toward the periphery areas at $1 \mathrm{~h}$ after cell seeding. At $24 \mathrm{~h}$, cells were fully attached and exhibited a polygonal shape, growing individually or clumping together (Fig. 1A). Immunostaining 


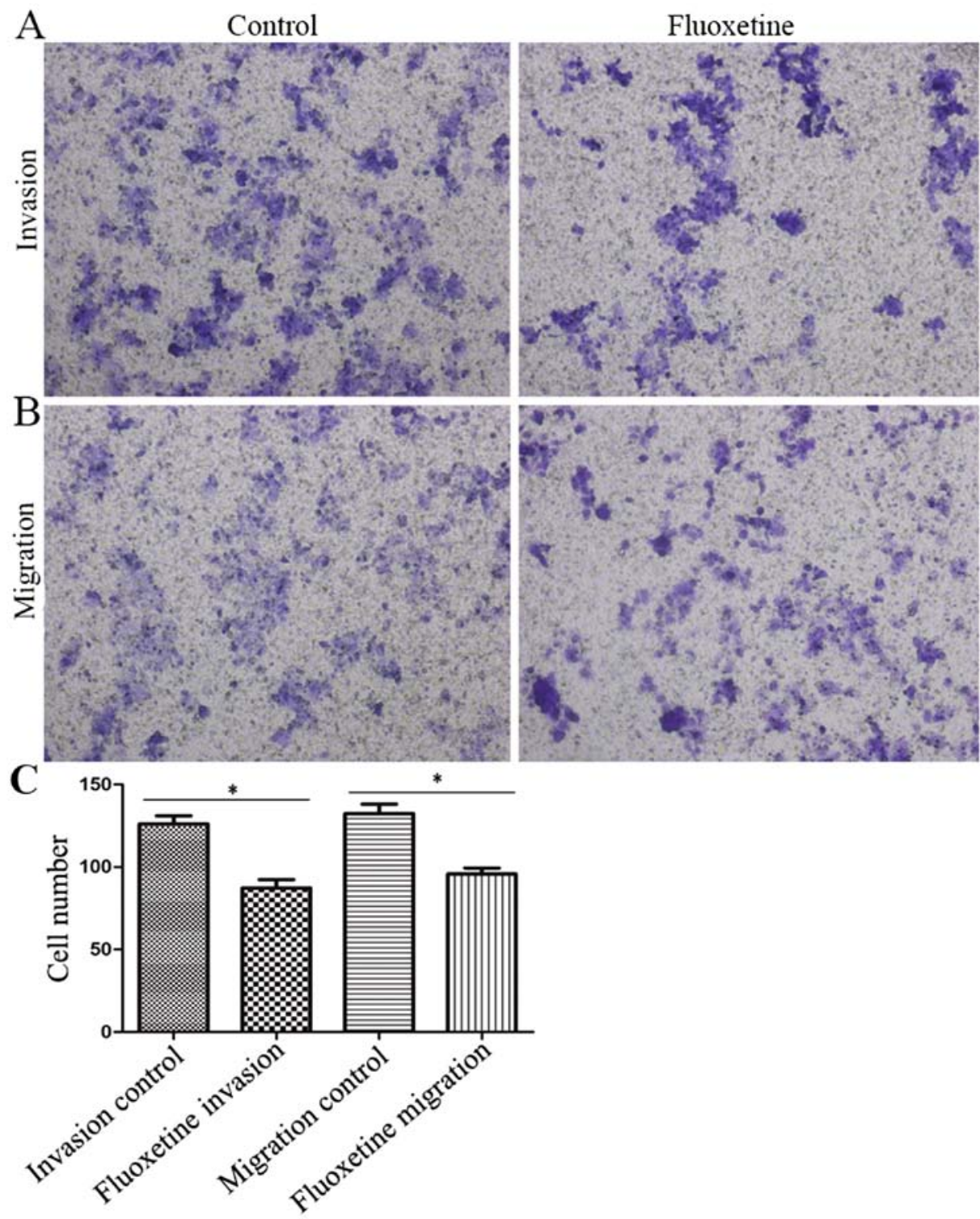

Figure 3. Fluoxetine treatment decreases the invasion and migration capability of HConEpiCs. (A) Images of invaded HConEpiCs untreated or treated with $5 \mu \mathrm{M}$ fluoxetine for $24 \mathrm{~h}$ (magnification x100). (B) Images of migrating HConEpiCs untreated or treated with $5 \mu \mathrm{M}$ fluoxetine for $24 \mathrm{~h}$ (magnification x100). (C) Quantification of invaded or migrated cells in A and B. " $\mathrm{P}<0.05$. HConEpiCs, human conjunctival epithelial cells.

assays demonstrated that most of the cells were positive for CK-13 staining, which indicated that the isolated cells were HConEpiCs (Fig. 1B).

Effect of fluoxetine on the viability of HConEpiCs. The results of the CCK-8 assay revealed that after $24 \mathrm{~h}$ of treatment with a series of fluoxetine dosages $(0,1,2.5,5,10,20$ and $40 \mu \mathrm{M})$, the viability of HConEpiCs gradually decreased. A slight decline in cell viability was observed when cells were exposed to fluoxetine at $1,2.5$ or $5 \mu \mathrm{M}$, with viabilities of $93.62 \pm 3.12$, $90.25 \pm 3.26$ and $86.28 \pm 3.42 \%$ of the control group, respectively. The cell viability markedly declined when cells were exposed to fluoxetine at 10,20 or $40 \mu \mathrm{M}$, with viabilities of $73.53 \pm 3.37,54.32 \pm 2.87$ and $39.33 \pm 4.06 \%$, of the control group, respectively. These results indicated that fluoxetine at $\geq 10 \mu \mathrm{M}$ exerted an evident cytotoxic effect on HConEpiCs. Therefore, $5 \mu \mathrm{M}$ fluoxetine was applied in the subsequent experiments (Fig. 2).
Fluoxetine inhibits cell migration and invasion. Transwell assays were performed to assess the effect of fluoxetine on the invasive and migratory capacity of HConEpiCs (Fig. 3). The invasion assay indicated that the number of cells that penetrated the Matrigel-coated membrane significantly decreased in the $5 \mu \mathrm{M}$ fluoxetine group, with the invasion capacity decreased to $69.31 \%$ of that in the control group (Fig. 3A and C). Furthermore, the Transwell migration assay demonstrated that the rate of cells that penetrated the membrane in the fluoxetine-treated group was significantly reduced, with the migration capacity decreased to $72.29 \%$ relative to that in the control group (Fig. 3B and C).

Expression of ERK1/2 and p-ERK1/2 in HConEpiCs via immunostaining and the effect offluoxetine on their expression. As indicated in Fig. 4, total ERK and p-ERK were widely abundant in the cytoplasm of HConEpiCs. However, HConEpiCs treated with $5 \mu \mathrm{M}$ fluoxetine for $24 \mathrm{~h}$ exhibited 

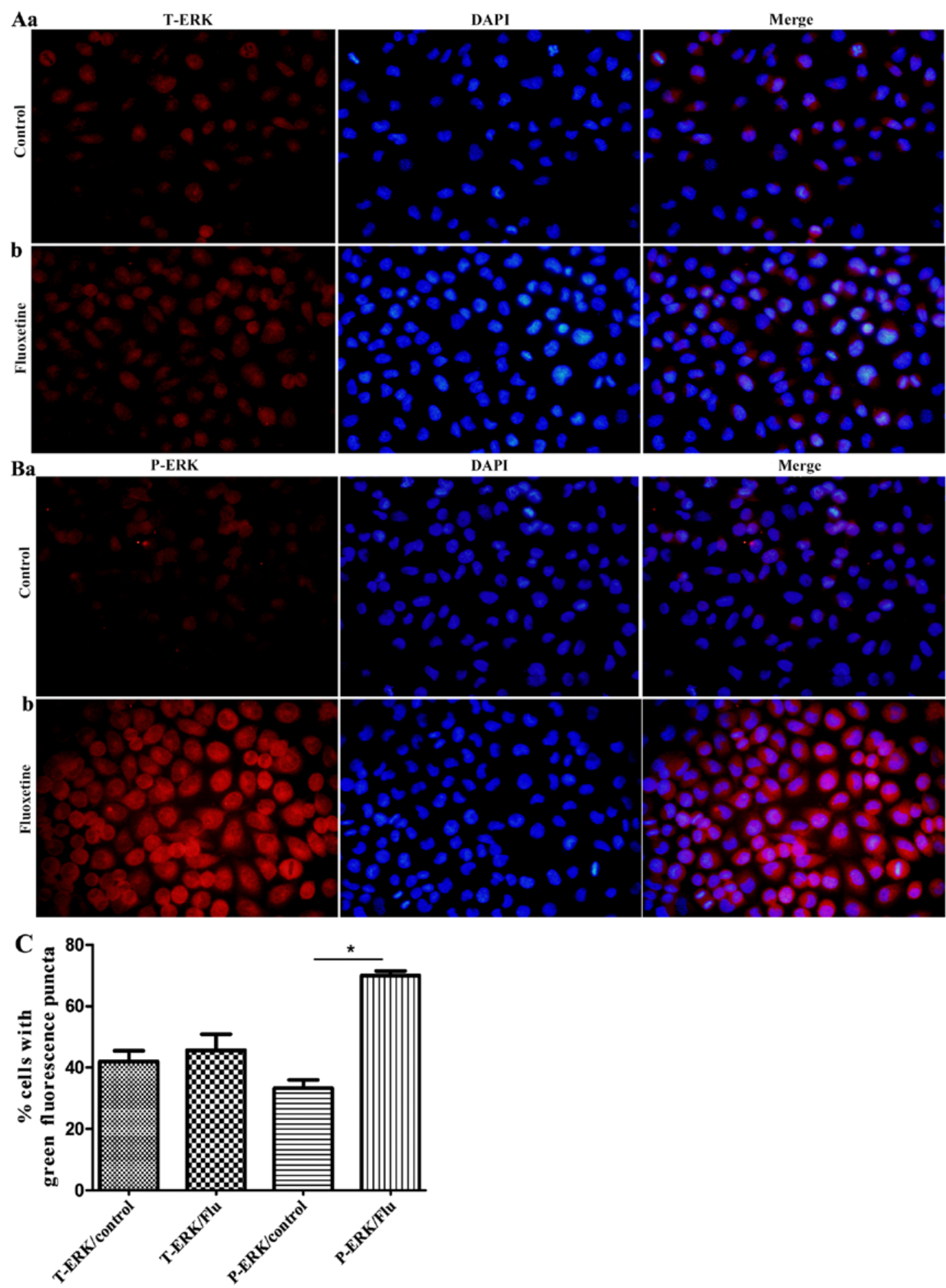

Figure 4. Immunostaining for(A) T-ERK and (B)P-ERK in human conjunctival epithelial cells without treatment (Aa,Ba control) or treated with $5 \mu \mathrm{M}$ fluoxetine for $24 \mathrm{~h}(\mathrm{Ab}, \mathrm{Bb})$ (red; magnification, $\mathrm{x} 200$; the DNA was counterstained with DAPI in blue). (C) Quantified levels of T-ERK and P-ERK.T/P-ERK, total/ phosphorylated ERK; Flu, fluoxetine. "P<0.05.

a notably increased protein level of p-ERK, while the level of total ERK did not significantly change.

Effect of fluoxetine on the protein levels of ERK1/2, p-ERK1/2, Bax, Bcl-2 and MMPs in HConEpiCs. HConEpiCs were treated with a gradient of fluoxetine doses $(0,1,2.5,5,10,20$ and $40 \mu \mathrm{M}$ ) for $24 \mathrm{~h}$ and their protein lysates were subjected to western blot analysis to assess the protein levels of ERK,
p-ERK, Bax, Bcl-2 and MMPs (Fig. 5). A representative western blot image is provided in Fig. 5A. The quantification results revealed that the protein levels of total ERK and MMP-2/9 were not significantly different between the fluoxetine-treated groups and the control (Fig. 5B and D). However, significantly elevated protein levels of $\mathrm{p}$-ERK and Bax were observed as the fluoxetine concentration increased and the maximum p-ERK level was achieved when fluoxetine was increased to $20 \mu \mathrm{M}$. 
A

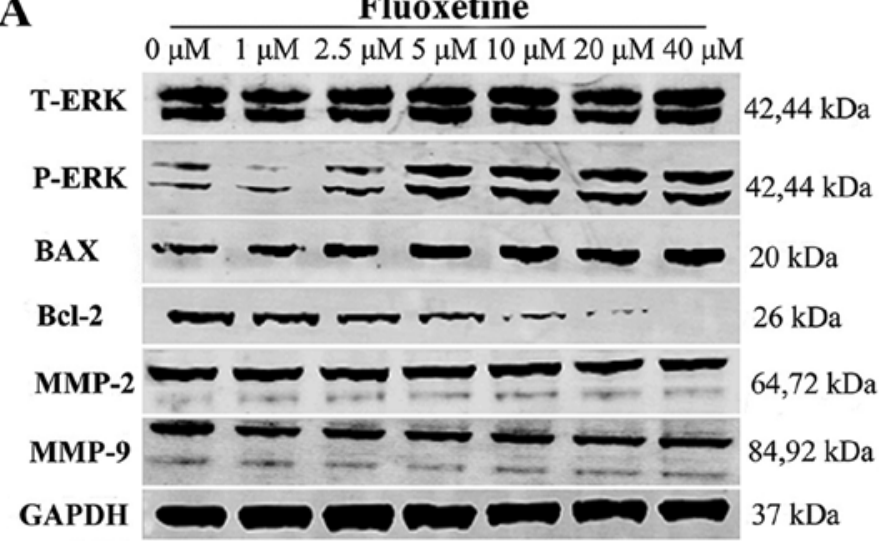

C

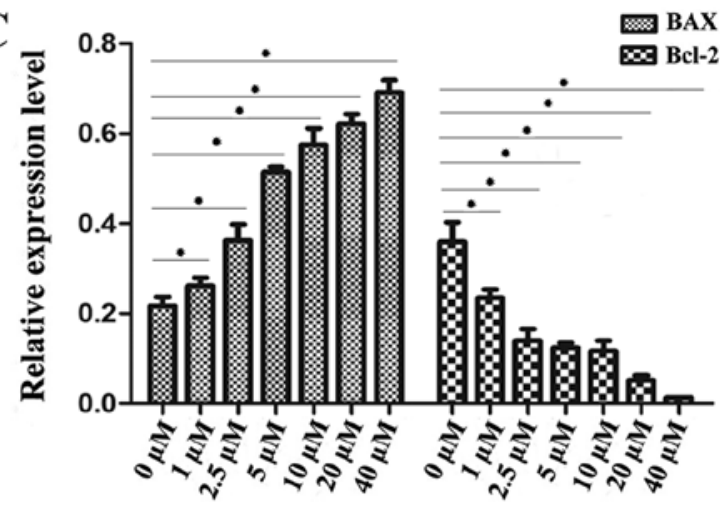

Concentration

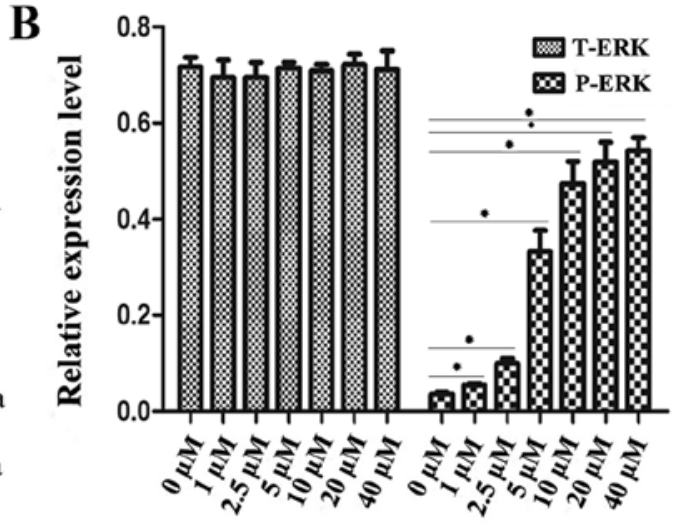

Concentration

D

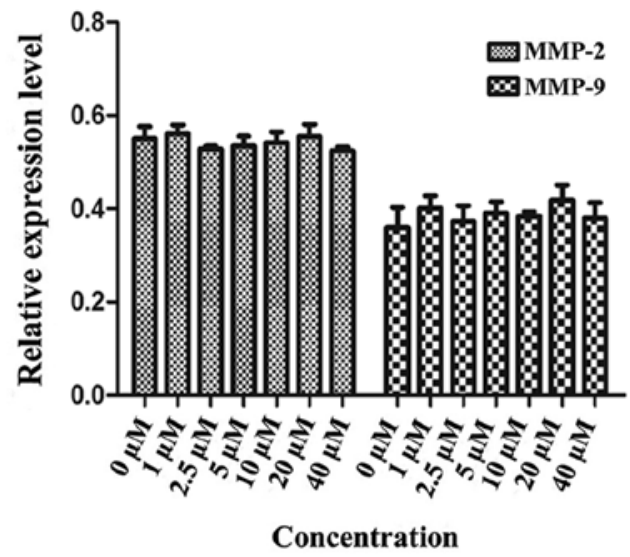

Figure 5. Expression levels of proteins associated with the MAPK-ERK1/2 pathway, apoptosis and MMPs in HConEpiCs treated by fluoxetine. (A) The protein levels of T-ERK, P-ERK, BAX, Bcl-2, MMP-2, MMP-9 and GAPDH in HConEpiCs treated by fluoxetine at different concentrations (0, 1, 2.5, 5, 10, 20 and $40 \mu \mathrm{M}$ ). (B) Quantification of protein levels of T-ERK and P-ERK in HConEpiCs prior to and after treatment with fluoxetine at a gradient of concentrations for $24 \mathrm{~h}$. (C) Quantification of protein levels of BAX and Bcl-2 in HConEpiCs prior to and after the 24-htreatment with fluoxetine at a gradient of concentrations. (D) Quantification of the protein levels of MMP-2 and MMP-9 in HConEpiCs prior to and after the 24-htreatment with fluoxetine at different concentrations. ${ }^{*} \mathrm{P}<0.05$. HConEpiCs, human conjunctival epithelial cells; MMP, matrix metalloproteinase; T/P-ERK, total/phosphorylated ERK.

Furthermore, the fluoxetine-treated groups exhibited significantly lower levels of Bcl-2 in comparison with the control group (Fig. 5B and C).

\section{Discussion}

MAPKs are highly conserved among eukaryotic cells and have significant roles in signal transduction. They participate in a variety of physiological and pathological processes, including cell proliferation, differentiation, apoptosis, stress responses and inflammatory responses (14). MAPK pathways maybe triggered by diverse exogenous cytokines or environmental factors, including hormones (insulin), growth factors (platelet-derived factors), inflammatory factors and TNFs, and behave as critical pathways that transduce the signal from the cell surface to the nucleus (14). There are three major MAPK family members: ERK, c-Jun N-terminal kinase and p38MAPK (14). The MAPK-ERK signaling pathway is able to amplify extracellular signals and has been the most widely studied pathway in recent years.

Fluoxetine is an SSRI that is frequently administered as an anti-depressant drug. Fluoxetine selectively inhibits 5-HT re-uptake by presynaptic membrane 5-HT pumps in neurons, thus increasing the concentration of 5-HT in the synaptic space and enhancing the biological functions of 5-HT pumps. Dwivedi et al (15) reported that the activity of ERK1/2 was significantly lower in brain tissues from patients who died from depression-associated suicide. 5-HT receptors are able to activate the MAPK pathway via phosphorylation, while anti-depressants may antagonize depression by manipulating activation of the MAPK pathway $(11,16)$. In addition, long-term treatment for depression may cause an intracellular increase of acute and chronic inflammatory factors, including IL- $\beta$ and TNF- $\alpha$, which may cause inflammation on the eye surface and promote dry eye syndrome (16).

In the present study, it was observed that the proliferation rates of HConEpiC gradually decreased in the presence of increasing dosages of fluoxetine. A dosage of $5 \mu \mathrm{M}$ was used in subsequent experiments. The results indicated that the phosphorylation of ERK was enhanced in the fluoxetine-treated group compared with that in the control group, while no significant difference was observed in the protein levels of total ERK. Furthermore, the protein levels of Bax and Bcl-2 were respectively upregulated and downregulated in the fluoxetine-treated group. These results suggest that fluoxetine at certain concentrations is able to activate the MAPK-ERK signaling pathway via triggering the phosphorylation of ERK1/2, subsequently causing apoptosis. In turn, this increase 
in cell death may further stimulate the MAPK-ERK pathway, establish a positive feedback loop of signaling transduction and resulting in dry eye syndrome. Bax and Bcl-2 are well-studied apoptosis-associated genes. Bax is a pro-apoptotic factor among Bcl-2 family proteins and its overexpression promotes cell death (17). By contrast, the Bcl-2 gene has an anti-apoptotic role during the process of programmed cell death and is widely accepted as an apoptosis inhibitor (17) Bcl-2 and Bax proteins also antagonistically form a heterodimer to regulate the progression of apoptosis.

According to previous studies, the ocular surface cells in patients with dry eye disease are abnormal with inflammatory cell infiltration, inducing the increase of inflammatory factors and the expression of MMPs. These studies indicate that desiccating stress stimulates the expression of MMP-9, IL- $1 \alpha$, IL- $1 \beta$ and TNF- $\alpha$ mRNA, as well as activates MAPK signaling pathways in the corneal epithelium. MAPKs are known to stimulate the production of inflammatory cytokines and MMPs, and they may have an important role in the induction of these factors that have been implicated in the pathogenesis of dry eye disease $(12,18)$. In the present study, after treatment of HConEpiCs with a gradient of fluoxetine concentrations for $24 \mathrm{~h}$, no significant difference in the protein expression levels of MMPs was observed, which was contrary to the expected outcome and requires further research.

In conclusion, fluoxetine at certain concentrations markedly induced apoptosis of HConEpiCs. The present study preliminarily examined the role of MAPK but due to the complexity of MAPK signaling pathway regulation, it was not possible to clarify the mechanism of 5-HT receptor increase in activating the MAPK-ERK pathway. The existence of 5-HT receptors in HConEpiCs is a limitation of the present study. 5-HT receptors exist in the neurons of brain tissues. Anti-depressant drugs may exert their effects by activating the MAPK-ERK pathway to then increase the concentration of 5-HT receptors. In the epithelial cells of the eye conjunctiva, anti-depressant drugs increase inflammatory cytokines and induce cell apoptosis by activating the MAPK-ERK pathway, resulting in dry eye conjunctiva. It may be speculated that HConEpiCs also express 5-HT receptor, and therefore, the present study does not reveal whether HConEpiCs express the 5-HT transporter, which may be replenished afterwards. Therefore, further study of the regulatory mechanism of the MAPK signal transduction pathway is required to identify proteins that directly regulate the MAPK-ERK pathway, and the association between apoptotic proteins and dry eye syndrome should be clarified. This will assist in developing drugs with better targeting abilities and fewer adverse reactions. The present study provided a foundation to investigate the mechanisms by which anti-depressant drug induces dry eye syndrome and may assist in developing novel therapies for dry eye syndrome.

\section{Acknowledgements}

Thanks is given to Professor Liao Hua from the Department of Otolaryngology Head and Neck Surgery, Renmin Hospital of Wuhan University, for his strong experimental technical support for this study. I would like to thank Mr Yan Jiangbo, a graduate student of ophthalmology at the school of medicine of Wuhan University, for his valuable experience in the preliminary experiments.

\section{Funding}

The present study received support from the Natural Science Foundation of Hubei province (grant no. 2016CKB708).

\section{Availability of data and materials}

The datasets used and/or analyzed during the current study are available from the corresponding author on reasonable request.

\section{Authors' contributions}

TC, who was a major contributor to the manuscript, participated in the design and operation of the experiment, and obtained, analyzed and interpreted the relevant data. YY participated in the conception and design of the experiment, and revised the contents of the manuscript. BC participated in the operation of the experiment and recorded the experimental process and data, while XW participated in the specific operations of the experiments and the drafting of the manuscript. All the authors have read and approved the final manuscript.

\section{Ethics approval and consent to participate}

The study was approved by the Clinical Research Ethics Committee of Renmin Hospital of Wuhan University (reference no. AF SOP/3.6-01/5.1). All patients in the study who provided disused conjunctival tissue blocks signed informed consent to the use of their tissue.

\section{Patient consent for publication}

Not applicable.

\section{Competing interests}

The authors declare that they have no competing interests.

\section{References}

1. Schiffman RM, Walt JG, Jacobsen G, Doyle JJ, Lebovics G and Sumner W: Utility assessment among patients with dry eye disease. Ophthalmology 110: 1412-1419, 2003.

2. Han SB, Hyon JY, Woo SJ, Lee JJ, Kim TH and Kim KW: Prevalence of dry eye disease in an elderly Korean population. Arch Ophthalmol 129: 633-638, 2011.

3. Lin PY, Cheng CY, Hsu WM, Tsai SY, Lin MW, Liu JH and Chou P: Association between symptoms and signs of dry eye among an elderly Chinese population in Taiwan: The shihpai eye study. Invest Ophthalmol Vis Sci 46: 1593-1598, 2005.

4. Management and therapy of dry eye disease: Report of the management and therapy subcommittee of the international dry eye WorkShop. Ocul Surf 5: 163-178, 2007.

5. Luo C, Wang F, Qin S, Chen Q and Wang QK: Coronary artery disease susceptibility gene ADTRP regulates cell cycle progression, proliferation, and apoptosis by global gene expression regulation. Physiol Genomics 48: 554-564, 2016.

6. Paul A, Wilson S, Belham CM, Robinson CJ, Scott PH, Gould GW and Plevin R: Stress-activated protein kinases: Activation, regulation and function. Cell Signal 9: 403-410, 1997. 
7. Pflugfelder SC, de Paiva CS, Tong L, Luo L, Stern ME and Li DQ: Stress-activated protein kinase signaling pathways in dry eye and ocular surface disease. Ocul Surf 3 (4 Suppl): S154-S157, 2005.

8. Li DQ, Luo L, Chen Z, Kim HS, Song XJ and Pflugfelder SC: JNK and ERK MAP kinases mediate induction of IL-1beta, TNF-alpha and IL-8 following hyperosmolar stress in human limbal epithelial cells. Exp Eye Res 82: 588-596, 2006.

9. Tang $\mathrm{CH}$ and Tsai CC: CCL2 increases MMP-9 expression and cell motility in human chondrosarcoma cells via the Ras/Raf/MEK/ERK/NF- $\kappa$ B signaling pathway. Biochem Pharmacol 83: 335-344, 2012.

10. Chang F, Steelman LS, Shelton JG, Lee JT, Navolanic PM, Blalock WL, Franklin R and McCubrey JA: Regulation of cell cycle progression and apoptosis by the Ras/Raf/MEK/ERK pathway (Review). Int J Oncol 22: 469-480, 2003.

11. Raison CL, Capuron L and Miller AH: Cytokines sing the blues: Inflammation and the pathogenesis of depression. Trends Immunol 27: 24-31, 2006.

12. Luo L, Li DQ, Doshi A, Farley W, Corrales RM and Pflugfelder SC: Experimental dry eye stimulates production of inflammatory cytokines and MMP-9 and activates MAPK signaling pathways on the ocular surface. Invest Ophthalmol Vis Sci 45: 4293-4301, 2004.

13. Dota A, Nishida K, Adachi W, Nakamura T, Koizumi N, Kawamoto S, Okubo K and Kinoshita S: An expression profile of activegenes in human conjunctival epithelium. Exp Eye Res 72: 235-241, 2001.
14. Krishna $\mathrm{M}$ and Narang $\mathrm{H}$ : The complexity of mitogen-activated protein kinases (MAPKs) made simple. Cell Mol Life Sci 65: 3525-3544, 2008.

15. Dwivedi Y, Rizavi HS, Zhang H, Roberts RC, Conley RR and Pandey GN: Aberrant extracellular signal-regulated kinase (ERK)1/2 signalling in suicide brain: Role of ERK kinase 1 (MEK1). Int J Neuropsychopharmacol 12: 1337-1354, 2009.

16. Kim KW, Han SB, Han ER, Woo SJ, Lee JJ, Yoon JC and Hyon JY: Association between depression and dry eye disease in an elderly population. Invest Ophthalmol Vis Sci 52: 7954-7958, 2011.

17. Kroemer G: The proto-oncogene Bcl-2 and its role in regulating apoptosis. Nat Med 3: 614-620, 1997.

18. De Paiva CS, Corrales RM, Villarreal AL, Farley WJ, Li DQ, Stern ME and Pflugfelder SC: Corticosteroid and doxycycline suppress MMP-9 and inflammatory cytokine expression, MAPK activation in the corneal epithelium in experimental dry eye. Exp Eye Res 83: 526-535, 2006.

This work is licensed under a Creative Commons Attribution-NonCommercial-NoDerivatives 4.0 International (CC BY-NC-ND 4.0) License. 